\title{
Electrical and Hall Effect Study of Hybrid Solar Cell
}

\author{
N. A. Nik Aziz, M. I. N. Isa, and S. Hasiah
}

\begin{abstract}
This work focuses on electrical and Hall Effect study of hybrid solar cell (HSCs). In particular, attention is given to investigations of HSCs with the architecture combining conjugated p-type polymer, poly (3-hexylthiophene) (P3HT), and inorganic $\mathrm{ZnO}$ heterojunctions with chlorophyll (CHLO) from marine microalgae as a dye. The films were prepared by spin coating technique and analyzed by using four point probes to calculate the conductivity. The results show that, the conductivity was increased by the increment of light intensity and concentration of CHLO. Lastly, the samples were analyzed using Hall Effect measurement to calculate the highest charge carrier in the sample of hybrid solar cell.
\end{abstract}

Index Terms-Chloropyhll, hybrid solar cell, poly (3-hexylthiophene), zinc oxide.

\section{INTRODUCTION}

Photovoltaics (PV), is a solar power technology that uses solar cells to convert incident sunlight directly into electricity with zero emissions. By now, PV technology has established itself as one of the best solutions to bring forth flexible and long-term solutions for rural electrification in the poorest areas of the world. Hybrid solar cell (HSC) is the combination of both organic and inorganic semiconductors. The HSC is also known as dye- sensitized solar cell which have an interesting low cost alternatives to conventional solar cell and their efficiencies is over $10 \%$ percent has been achieved [1].HSC combine advantages of both organic and inorganic semiconductors. Hybrid photovoltaics have organic materials that consist of conjugated polymers that absorb light as the donor and transport holes [2].The term organic semiconductor is used to describe organic materials (conjugated oligomers or polymers) that possess the ability of transporting charge carriers and have been studied since the 1950s [3]. The electronic conductivity of these materials lies between that of metals and insulators spanning a broad range between $10^{-7}$ and $10^{3} \mathrm{Scm}^{-1}$. Holes and electrons in $\pi$ orbitals are the typical charge carriers in organic semiconductors. Charge transport typically depends on the ability of the charge carriers to move from one molecule to another, which depends on the energy gap between highest occupied molecular orbital (HOMO) and lowest unoccupied molecular orbital (LUMO) levels. These materials are either based on oligomers such as pentacene, anthracene, rubrene, or oligothiophenes, or on polymers such as polypyrrole,

Manuscript received August 21, 2013; revised December 18, 2013. This work was supported in part by Ministry of Higher Education for Exploratory Research Grant Scheme (ERGS-Vot: 55065).

N. A. Nik Aziz and S. Hasiah are with the Renewable Energy Research Interest Group, Universiti Malaysia Terengganu, Malaysia 21030 (e-mail: nikaznikal.umt@gmail.com, hasiah@umt.edu.my).

M. I. N. Isa is with the Advanced Material Research Group, Universiti Malaysia Terengganu, Malaysia 21030 (e-mail: ikmar_isa@umt.edu.my). polyacetylene, poly (3-hexylthiophene) (P3HT), or poly (p-phenylenevinylene) (PPV). Organic semiconductors have attracted much attention because of their fundamental scientific importance and impressive improvements in performance in a wide variety of photonic applications, such as organic light-emitting diodes (OLED), organic field-effect transistors (OFET), organic solar cells (OSC), liquid crystals, sensors, and many more[4]-[7]. Inorganic materials in hybrid cells are used as the acceptor and electron transporter in the structure. The hybrid photovoltaic devices have a potential for not only low-cost by roll-to-roll processing but also for scalable solar power conversion. In hybrid solar cells, an organic material is mixed with a high electron transport material to form the photoactive layer [8]. The two materials are assembled together in a heterojunction-type photoactive layer, which can have greater power conversion efficiency than a single material [9].

In this study, a hybrid solar cell were formed to find its conductivity, Hall Effect measurement and to elucidate its efficiency in the absorption of energy. Zinc oxide and a polymer, P3HT were used together with CHLO in producing this hybrid solar cell. $\mathrm{ZnO}$, a wide band gap inorganic semiconductor has high crystalinity and conductivity in photoelectric properties, and it was prepared by hydrothermal method [10]. The nature dye was used as sensitizers because of sensitizers having a broad absorption band in conjunction with oxide films of nanocrstalline morphology permits to harvest a large fraction of sunlight [11].

\section{EXPERIMENTAL PROCEDURES}

\section{A. Materials}

Poly (3-hexylthiophene) was synthesized before used. Zinc oxide $(\mathrm{ZnO})$ was synthesized by using hydrothermal method and left to growth on the indium tine oxide (ITO) coated glass. The natural dye was synthesized from marine microalgae to get the chlorophyll. The others raw materials such as methanol, acetone, zinc acetate dehydrate,zinc nitrate and hexamethylenetetramine were purchased from Sigma Aldrich.

\section{B. Sample Preparation}

\section{1) Cleanup the ITO coated glass}

The ITO coated glass must be clean to make it free from dirt and dust and also to avoid any contamination. Cleanliness of ITO is very important to reduce any impurities present in the ITO because it will affect the accuracy of the results. Ultrasonic machine was used to clean the ITO coated glass. Firstly, ITO coated glass were immersed in the $20 \mathrm{ml}$ detergent solution for 10 minutes at $30^{\circ} \mathrm{C}$. Secondly, the ITO coated glasses were put in distilled water for 5 minutes at the same temperature. This step was repeated three times in order to clean the detergent solution on the ITO glass. Then, the 
step was continued with acetone for 5 minutes at $30^{\circ} \mathrm{C}$. Lastly, the ITO coated glasses were immersed with distilled water to remove stained of acetone for 5 minutes at $30^{\circ} \mathrm{C}$ and left to dry completely at room temperature.

\section{2) Fabrication of hybrid solar cell}

The samples were coated by spin coating techniqueto ensure the thin film was applied uniformly on the ITO coated glass. The fabrication of hybrid solar cell is shown in Fig. 1.

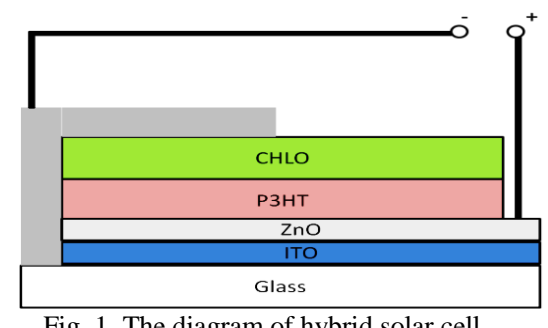

Fig. 1. The diagram of hybrid solar cell.

\section{Characterization and Measurements}

\section{1) Electrical conductivity}

Electrical conductivity is the capacity of any object or substance to conduct an electric current. When an electrical pottential difference is placed across a conductor its movable charges flow, giving rise to an electric current. The conductivity can be measured as follow [12]:

$$
R_{S}=4.532 \frac{V}{I}
$$

where,

$R_{\mathrm{S}}=$ sheet resistance (resistivity),

$4.532=$ correction factor,

$V=$ voltage measured and

$I=$ the current applied from the test unit.

Thus, electrical conductivity can be determined by;

$$
\sigma=1 / R_{S}
$$

where,

$\sigma=$ electrical conductivity and

$\mathrm{R}_{\mathrm{S}}=$ the sheet resistivity.

\section{2) Efficiency of hybrid solar cell}

The efficiency of hybrid solar cell can be calculated as follow,

$$
\begin{gathered}
\eta=\frac{P_{o u t}}{P_{\text {in }}} \times 100 \% \\
P_{\text {in }}=\text { Intensity } \times \text { Effective Surface Area } \\
P_{\text {out }}=I_{\text {max }} \times V_{\text {max }}
\end{gathered}
$$

where, $\eta$ is the efficiency, $I$ is current and $V$ is voltage.

\section{3) Hall effect measurement(HEM)}

In HEM, the samples should have well-defined geometries and good ohmic contacts in order to obtain the accurate results. The samples must have vdP geometry. The ITO substrates were placed on the sample holder as shown in Fig. 2. The sample on the holder system is then connected to contacts 1, 2, 3 and 4, using the silver paint on four edges.

The connection is then tested using a multimeter to measure the resistance for each contact to ensure proper contact.

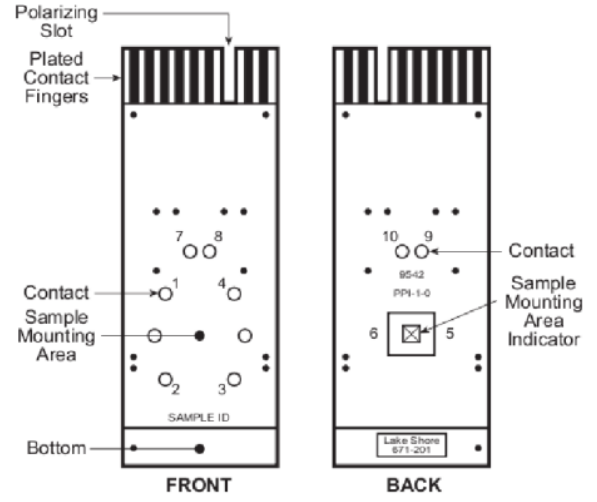

Fig. 2. The geometry of sample holder.

The measurements were performed using the Leois-JSF software which developed and tested through the corporation of Physical Sciences Department, Universiti Malaysia Terengganu, lead by Dr Salleh Harun and Nanorian Technology [13]. The Hardware system called Hall Effect Measurement (HEM) system model 7600 is supplied by Lakeshore Ltd. The important part of this HEM system is ensuring that the room temperature and set temperature was equivalent $\left(20^{\circ} \mathrm{C}\right)$ in order to prevent the power supply from breakdown. The measurement consists of two parts. The first parts are called the IV curve traces measurement and the second part is variable magnetic field measurement. The purpose of IV curve traces measurement is to make sure that all the contacts are in good connections. In this work, the magnetic field fixed was $10 \mathrm{kG}$ (1 Tesla) and the current was $0.1 \mathrm{~A}$.

In addition, Fig. 3 shows the numbering of sample for Hall calculations which used in this work.

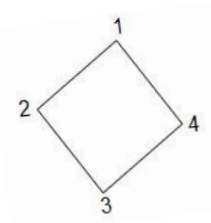

Fig. 3. The numbering of sample for Hall calculations

The explanations below are according to Fig. 3 .

1) $V_{+31,42(+B)}=$ The current is passed from point 3 to 1 , and the Hall voltage measured between 4 and 2 .

2) $V_{-31,42(+B)}=$ The current is passed from point 1 to 3 , and the Hall voltage measured between 4 and 2 .

3) $V_{+42,13(+B)}=$ The current is passed from point 4 to 2 , and the Hall voltage measured between 1 and 3 .

4) $V_{-42,13(+B)}=$ The current is passed from point 2 to 4 , and the Hall voltage measured between 1 and 3 .

5) $V_{+31,42(-B)}=$ The current is passed from point 3 to 1 , and the Hall voltage measured between 4 and 2 in negative field.

6) $V_{-31,42(-B)}=$ The current is passed from point 1 to 3 , and the Hall voltage measured between 4 and 2 in negative field.

7) $V_{+42,13(-B)}=$ The current is passed from point 4 to 2 , and the Hall voltage measured between 1 and 3 in negative field.

8) $V_{-42,13(-B)}=$ The current is passed from point 4 to 2 , and the Hall voltage measured between 1 and 3 in negative field. According to [13], HEM can be callculted as follow, 
Hall voltage average, $V_{H \text { avg }}$;

$=\left[V_{+31,42(+B)}-V_{+31,42(-B)}+V_{-31,42)-B)}-V_{-31,42(+B)}\right.$

$\left.-V_{+42,13(-B)}+V_{-42,13(-B)}-V_{-42,13(+B)}\right] / 8$

For Hall coefficient $\left(R_{H}\right)$ in unit $\mathrm{cm}^{3} . \mathrm{C}^{-1}$, two values of $R_{H}$ are calculated using following equations;

$$
\begin{gathered}
R_{H C}=10^{8} \frac{t[\mathrm{~cm}]}{B[G]} \\
+V_{-31,42(-B)}-V_{+31,42(-B)} \\
+I_{-31(-B)}-I_{+31(-B)}
\end{gathered}
$$

and

$$
\begin{array}{r}
R_{H D}=10^{8} \frac{t[\mathrm{~cm}]}{B[G]} \cdot \frac{V_{+42,13(+B)}-V_{-42,13(+B)}}{I_{+42(+B)^{-I}-42(+B)}} \\
\frac{+V_{-42,13(-B)}-V_{+42,13(-B)}}{+I_{-4(-B)}-I_{+42(-B)}}
\end{array}
$$

The Hall coefficient average, $R_{H \text { avg }}$ calculated by;

$$
R_{H \text { avg }}=\frac{R_{H C}+R_{H D}}{2}
$$

If the thickness is unknown, the layer or sheet carrier concentration, $n_{s}=n$, is used instead of the bulk density,

$$
n_{s}=\frac{8 \times 10^{-8} \times I B}{q v_{H(t o t a l)}} \mathrm{cm}^{-2}
$$

where, $B$ in Gauss unit, $I$ in ampere, and $V_{H}$ in volt. Then the Hall mobility $\left(\mu_{H}\right)$ is given by

$$
\mu_{H}=\frac{\mid V_{H} \text { avg } \mid}{\rho_{\text {avg }}}\left[\mathrm{cm}^{2} \cdot V^{-1} \cdot \mathrm{s}^{-1}\right]
$$

Lastly, types of charge carrier. It is determined by the polarity sign of $V_{H \text { avg }}$ from (6) and polarity sign of $R_{H \text { avg }}$ in (9). If the polarity sign is positive, the type of charge carrier is holes and called P-type. In contrast, if negative sign, it is electrons and called n-type.

\section{RESULT AND DISCUSSION}

\section{A. Conductivity Study}

The conductivity at different intensity of light is shown in the Fig. 4. The conductivity was proportional with the intensity of light for sample 5-CHLO and 10-CHLO with the regression value 0.99 and 0.98 respectively. The conductivity of the sample with and without CHLO shows the different because of CHLO are involved in photosystem assembly and contribute to light harvesting by absorbing light energy in a region of the visible spectrum. It also provides protection from excess light via energy dissipation and free radical detoxification. Thus, the conductivity of the sample with CHLO is more stable.

The temperature-dependent conductivity measurements are carried out to analyze the mechanism of ionic conduction in hybrid solar cell. Fig. 5 shows the variation ionic conductivity with the reciprocal temperature for hybrid solar cell. The linear variation of logs versus $1000 / \mathrm{T}$ plots for sample 5-CHLO and 10-CHLO suggests an Arrhenius type thermally activated process. This suggested that there is no phase transition of hybrid materials in the temperature range studied.

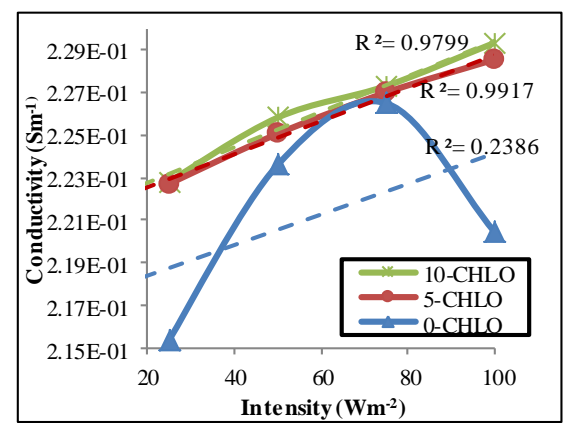

Fig. 4. The conductivity at different intensity of light.

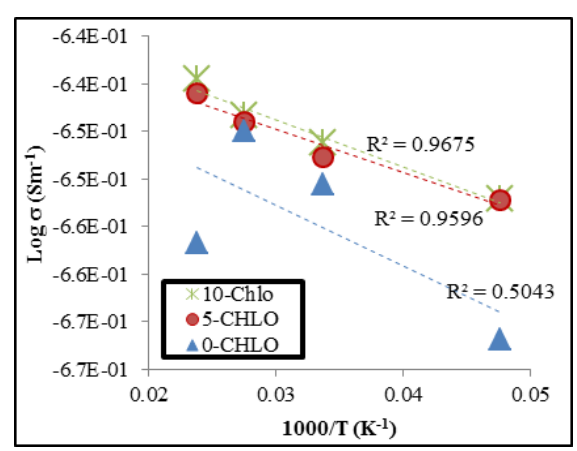

Fig. 5. Temperature dependent for the samples.

The conductivity can be expressed

$$
\sigma=\sigma_{o} \exp \left(-\frac{E_{a}}{k T}\right)
$$

$$
\text { Intensity of light }=k T^{4}
$$

where the $\sigma_{o}$ is the pre-exponential factor, $E_{a}$ is activation energy, $\mathrm{k}$ is Boltzmann constant $\left(5.678 \mathrm{E}-8 \mathrm{Wm}^{-2}-\mathrm{K}^{4}\right)$ and $\mathrm{T}$ is temperature.

Due to the black body radiation, the black body at a specific temperature is determines by the intensity of light at each frequency as shown in equation (13). Table I shows the activation energy of blended polymer films with different composition. $E_{a}$ can be obtained from the slope and the pre-exponential factor can be obtained from the intercept at the vertical axis from the Fig. 5 [14]. The results reveal that $E_{a}$ decreases effectively with the increasing of conductivity. From these results, interaction of polar molecules of the hybrid materials may help to dissociate the $\mathrm{H}^{+}$ions in the CHLO. As temperature increases, the polymer chain acquires faster internal modes in which bond rotations produce segmental motion. This, in turn, favors hopping inter-chain and intra-chain ion movements and, accordingly, the conductivity of the polymer electrolyte becomes high [15].

TABLE I: CONDUCTIVITY AND ACTIVATION ENERGY OF THE SAMPLES AT 100WM-2

\begin{tabular}{|c|c|c|}
\hline Samples & $\begin{array}{c}\text { Conductivity } \\
\times 10^{-1}\left(\mathrm{Sm}^{-1}\right)\end{array}$ & $\begin{array}{c}\text { Activation } \\
\text { Energy (eV) }\end{array}$ \\
\hline 0-CHLO & 2.204 & 3.270 \\
\hline 5-CHLO & 2.286 & 4.216 \\
\hline 10-CHLO & 2.294 & 4.547 \\
\hline
\end{tabular}




\section{B. Efficiency of Hybrid Solar Cell}

Fig. 6 shows the efficiency of the samples at different intensity of light. Again, the sample with CHLO shows the highest efficiency with $0.1 \%$ to $0.4 \%$. These results were significant as the results in the Fig. 4 and Fig. 5 respectively. The interaction between $\mathrm{ZnO}$ and the CHLO plays an important role towards the efficiency of HSC. According to [16], this might be due to possible inefficient electron/dye cation recombination pathways.

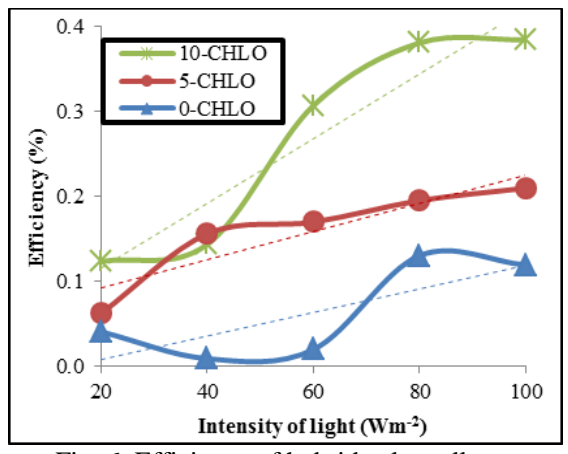

Fig. 6. Efficiency of hybrid solar cell.

In fact $\mathrm{H}^{+}$are the potential determining ions for $\mathrm{ZnO}$ and that proton adsorption causes a positive shift of the Fermi level of the $\mathrm{ZnO}$, thus limiting the maximum photovoltage that could be delivered by the cells.

Thus, introducing a functional group, such as carboxyl group and optimizing the structure of CHLO are necessary to improve the efficiency HSC. Some complication such as dye aggregation on nanocrystalline film produces absorptivity that results in no electron injection. According [17], dye aggregation is a serious issue that occurs when compounds fill the free space between the dye molecules, partially blocking the physical contact between the P3HT and $\mathrm{ZnO}$ semiconductor film surface, reducing reaction and inhibiting dye aggregation.

\section{Hall Effect Study}

The mobile charges in conventional conducting materials are negatively charged (they are, in fact, electrons). Hall Effect can be used to determine whether the mobile charges in materials are positively or negatively charged.

\section{1) Hall voltage and hall coefficient}

Clearly, it is possible to determine the sign of the mobile charges in a current carrying conductor by measuring the Hall voltage.

\begin{tabular}{ccc}
\multicolumn{2}{c}{ TABLE II: HALL VOLTAGE OF MATERIALS IN HYBRID SOLAR CELL } \\
\hline \multirow{2}{*}{ Materials } & Hall Voltage, & Hall Coefficient, \\
& $\mathbf{V}_{\text {Hall }} \mathbf{x} \mathbf{1 0}^{-\mathbf{3}}(\mathbf{m} \mathbf{)})$ & $\mathbf{R}_{\mathbf{H}} \mathbf{x} \mathbf{1 0}^{-\mathbf{3}}\left(\mathbf{c m}^{-\mathbf{2}} \mathbf{V}^{-\mathbf{1}} \mathbf{S}^{\mathbf{- 1}}\right)$ \\
\hline ZnO & -2.132 & -2.193 \\
P3HT & -3.923 & -1.690 \\
CHLO & -1.552 & -1.628 \\
Hybrid & -2.120 & -2.211 \\
\hline
\end{tabular}

The results in the Table II show the value of hall voltage and hall coefficient for materials in the hybrid solar cell. The value is negative for all materials and it is show that the mobile charges are negative. This charge is the same with the metals (because they are electrons). However, in some types of semiconductor the mobile charges turn out to be positive. These positive charge carriers are called holes. Holes are actually missing electrons in the atomic lattice of the semiconductor, but they act essentially like positive charges.

\section{2) Carrier concentration and hall mobility}

The excitation of a carrier from the valence band to the conduction band creates free carriers in both bands. The concentration of these carriers is called the intrinsic carrier concentration, denoted by $\mathrm{n}_{\mathrm{s}}$. Semiconductor material which has not had impurities added to it in order to change the carrier concentrations is called intrinsic material. The intrinsic carrier concentration is the number of electrons in the conduction band or the number of holes in the valence band in intrinsic material. This number of carriers depends on the band gap and on the situation of the material such as temperature.

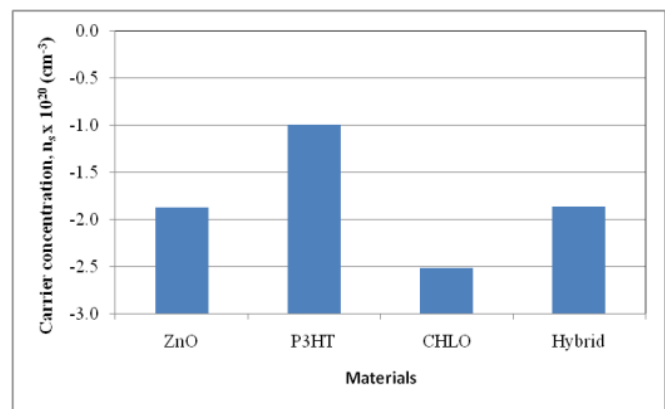

Fig. 7. Charge carrier concentration of materials in hybrid solar cell.

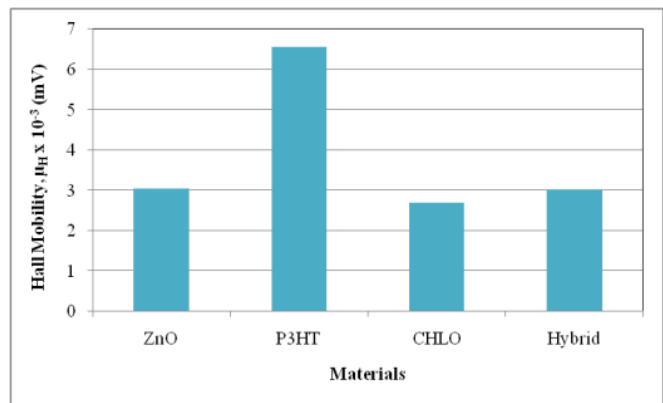

Fig. 8. Hall mobility of materials in hybrid solar cell.

Fig. 7 and Fig. 8 show the charge carrier concentration and hall mobility of materials in the hybrid solar cell. From both of Figure, P3HT show the highest value of charge carrier concentration and hall mobility followed by hybrid solar cell, $\mathrm{ZnO}$ and CHLO. The energy band gap for P3HT is $1.9 \mathrm{eV}$, $\mathrm{ZnO}$ is $3.37 \mathrm{eV}$ and for CHLO is $5.15 \mathrm{eV}$. A large band gap is more difficult for a carrier to be excited across the band gap, and therefore the charge carrier concentration is lower in higher band gap materials. Alternatively, increasing the temperature makes it more likely that an electron was excited into the conduction band, which increased the charge carrier concentration. The larger free carrier density is due to the low resistivity [18], [19] and high conductivity of film [20].

\section{CONCLUSION}

The organic/inorganic HSCs with the architecture combining conjugated p-type polymer, poly (3-hexylthiophene) (P3HT), and inorganic $\mathrm{ZnO}$ heterojunctions with chlorophyll (CHLO) from marine microalgae as a dye were successfully prepared by spin coating technique. The highest conductivity at $100 \mathrm{Wm}^{-2}$ was $2.294 \times 10^{-1} \mathrm{Sm}^{-1}$ for sample 10-CHLO. The sample 
10-CHLO also shows the highest efficiency with $0.1 \%$ to $0.4 \%$. From the Hall Effect measurement it was found that, polarity sign of $V_{H \text { avg }}$ and polarity sign of $R_{H \text { avg }}$ obtained were negative for all samples. This finding indicates that the majority carriers are electron.

\section{ACKNOWLEDGMENT}

The financial support from Ministry of Higher Education for Exploratory Research Grant Scheme (ERGS-Vot: 55065) is greatly acknowledged. The authors also would like to thank the Physical Sciences Department under the Faculty of Science and Technology, Universiti Malaysia Terengganu, for the help and support given for this work.

\section{REFERENCES}

[1] L. S. Mende, U. Bach, R. H. Baker, T. Horiuchi, H. Miura, S. Ito, and S. Uchida, "Organic dye for highly efficient solid-state dye-sensitized solar cells," Advanced Materials, vol. 17, no. 7, pp. 813-815, 2005.

[2] D. J. Milliron, I. Gur, and A. Alivisatos, "Hybrid organic-nanocrystal solar cells," MRS Bulletin, vol. 30, pp. 41-44, 2005.

[3] M. Pope and C. E. Swenberg, Electronic Processes in Organic Crystals and Polymers, $2^{\text {nd }}$ ed., New York: Oxford University Press, 1999.

[4] Z. Bao and Z. Locklin, Organic Field-Effect Transistors, New York: CRC, 2007.

[5] T. A. Skotheim, R. L. Elsenbaumer, and J. R. Reynolds, Handbook of Conducting Polymers, New York: CRC, 2007.

[6] C. Brabec, V. Dyakonov, and U. Scherf, Organic Photovoltaics: Materials, Device Physics, and Manufacturing Technologies, Weinheim: Wiley-VCH, 2008.

[7] I. F. Perepichka and D. F. Perepichka, Handbook of Thiophene-Based Materials: Applications in Organic Electronics and Photonics, Weinheim: Wiley-VCH, 2009.

[8] S. E. Shaheen, D. S. Ginley, and G. E. Jabbour, "Organic-based photovoltaics," MRS Bulletin, vol. 30, 2005, pp. 10.

[9] B. R. Saunders and M. L. Turner, "Nanoparticle-polymer photovoltaic cells," Advances in Colloid and Interface Science, vol. 138, no. 1, pp. $1-23,2008$.

[10] R. Thitima, C. Patcharee, S. Takashi, and Y. Susumu, "Efficient electron transfers in ZnOnanorod arrays with N719 dye for hybrid solar cells," Solid-State Electronics, vol. 53, no. 2, pp. 176-180, 2009.

[11] M. Grätzel, "Dye-sensitized solar cells," Journal of Photochemistry and Photobiology C. Photochemistry Reviews, vol. 4, no. 2, pp. 145-153, 2003.

[12] S. Hasiah, K. Ibrahim, H. B. Senin, and K. B. K. Halim, "Electrical conductivity of chlorophyll with polythiophene thin film on indium tin Oxide as $p-n$ Heterojunction Solar Cell," Journal of Physical Science, vol. 19, no. 2, pp. 77-92, 2008.

[13] A. R. N. Laily, M. I. N. Isa, H. Salleh, and S. Hasiah, "Hall effect of Polypyrole-Chlorophyl blend thin film: solar cell," Solid State Science and Technology, vol. 19, no. 2, pp. 107-119, 2011.

[14] S. R. Majid and A. K. Arof, "Proton-conducting polymer electrolyte films based on chitosan acetate complexed with $\mathrm{NH}_{4} \mathrm{NO}_{3}$ salt," Physica $B$, vol. 355, pp. 78-82, 2005.

[15] C. V. S. Reddy, A. K. Sharma, and V. V. R. N. Rao, "Conductivity and discharge characteristics of polyblend (PVP $\left.+\mathrm{PVA}+\mathrm{KIO}_{3}\right)$ electrolyte," Journal of Power Sources, vol. 114, issue 2, pp. 338-345, 2003.

[16] G. Calogero, G. D. Marco, S. Cazzanti, S. Caramori, R. Argazzi, and A. D. Carlo, "Efficient dye-sensitized solar cells using red turnip and purple wild Sicilian prickly pear fruits," International Journal of Molecular Sciences, vol. 11, pp. 254-267, 2010.

[17] G. Calogero and G. D. Marco, "Red Sicilian orange and purple eggplant fruits as natural sensitizers for dye-sensitized solar cells," Solar Energy Materials and Solar Cells, vol. 92, pp. 1341-1346, 2008.

[18] X. W. Sun, D. H. Kim, and H. S. Kwok, "Ultra thin ITO films deposited on various substrates by pulsed laser deposition," MRS Proceedings, vol. 485, pp. 267-272, Dec. 1997.

[19] S. J. Takayama, T. Sugawara, A. Tanaka, and T. Himuro, "Indium tin oxide films with low resistivity and low internal stress," Journal of Vacuum Science Technology A, vol. 21, no. 4, pp. 1351-1354, 2003.

[20] S. A. Bashar, "Study of Indium Tin Oxide (ITO) for novel optoelectronic devices," Ph.D. dissertation, Department of Electronic Engineering, University of London, 1997.

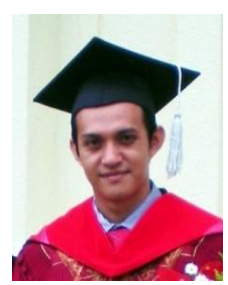

Nik Aziz Nik Ali was born in Setiu, Terengganu, Malaysia in 1984. He received his B.S. degree in physic electronics and instrumentation from Universiti Malaysia Terengganu, Malaysia, in 2007, and then received his M.S. degree in physics in 2010 and studying Ph.D. in Physics in Universiti Malaysia Terengganu, Malaysia, from 2010. This is the 3 year of his Ph.D. study. His current research interests include polymer electrolytes, hybrid solar cell and corrotion. Mr. Nik Aziz has published four SCI journals and attended 2013 international conference on alternative energy in developing countries and emerging economies held in Bangkok, Thailand. He also won Bronze Medal at Malaysian Technology Expo 2013, Invention \& Innovation Awards.

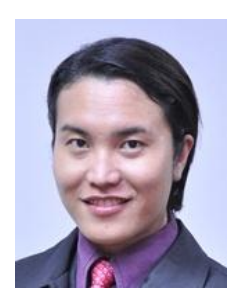

Mohammad Ikmar Nizam Mohd Isa was born in Kuala Lumpur, Malaysia in 1979. He received his B.S. degree in computational physics and Electronics from University of Malaya, Malaysia, in 2002 and the $\mathrm{Ph}$. D. degree from Department of Physics, University of Malaya in 2006. In 2007 at Universiti Malaysia Terengganu, associate professor Dr. Ikmar Sung worked as physics lecturer. Currently, he was appointed as head of physics department at faculty of science and technology, Universiti Malaysia Terengganu. His research has been focused on advanced material science. Since the energy issue is getting more and more important part of his research has been focused on the development of proton battery in the past 5 years.

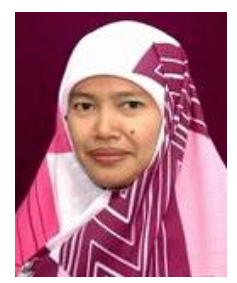

Hasiah Salleh was born in Terengganu, Malaysia in 1969. She received her B.S. degree in physic Nuclear Science from National University of Malaysia, Malaysia, in 1998, and then received her M.S. degree in physics in 2010 and studying Ph.D. in Physics in University of science Malaysia, Penang, Malaysia, from 2010. This is the 3rd year of her Ph.D. study. Currently, she is a lecturer at physical department at Universiti Malaysia Terengganu. Her current research interests include organic solar cell, conducting polymer and renewable energy. Mrs. Hasiah has published a few SCI journals and attended more than 15 international conferences during her study. He also won gold Medal and the best award at Malaysian Technology Expo 2013, Invention \& Innovation Awards. 